\title{
Manipulation of magnetic nanoparticle retention and hemodynamic consequences in microcirculation: assessment by laser speckle imaging
}

\author{
This article was published in the following Dove Press journal: \\ International Journal of Nanomedicine \\ 9 June 2012 \\ Number of times this article has been viewed
}

\author{
Yunn-Hwa Ma' \\ Si-Yi Chen' \\ Shu-Ju Tu \\ Hung-Wei Yang ${ }^{3}$ \\ Hao-Li Liu ${ }^{4}$ \\ 'Department of Physiology \\ and Pharmacology and Healthy \\ Aging Research Center, ${ }^{2}$ Department \\ of Medical Image and Radiological \\ Sciences, ${ }^{3}$ Department of \\ Chemical and Material Engineering, \\ ${ }^{4}$ Department of Electrical Engineering, \\ College of Medicine and College of \\ Engineering, Chang Gung University, \\ Kuei-Shan, Tao-Yuan, Taiwan, ROC
}

\begin{abstract}
Magnetic nanoparticles (MNPs) have been proposed for targeted or embolization therapeutics. How MNP retention occurs in circulation may critically determine local hemodynamics, tissue distribution of MNPs, and the therapeutic effects. We attempted to establish a microcirculation model to study the magnetic capture of MNPs in small vessels and to determine the factors affecting MNP retention. Two-dimensional hemodynamic changes in response to magnet-induced MNP retention in the microvessels of the cremaster muscle in vivo were observed in a real-time manner using a laser speckle imaging technique. Changes in tissue perfusion of the cremaster muscle appeared to be closely correlated with the location of the magnet placement underneath the muscle in response to intra-arterial administration of dextran-coated MNPs. Magnet-related retention was observed along the edge of the magnet, as corroborated by the results of histology analysis and microcomputed tomography. In these preparations, tissue iron content almost doubled, as revealed by inductively coupled plasma optical emission spectroscopy. In addition, MNP retention was associated with reduced downstream flow in a dose-dependent manner. Dissipation of MNPs $(5 \mathrm{mg} / \mathrm{kg})$ occurred shortly after removal of the magnet, which was associated with significant recovery of tissue flow. However, MNP dissipation did not easily occur after administration of a higher MNP dose $(10 \mathrm{mg} / \mathrm{kg})$ or prolonged exposure to the magnetic field. An ultrasound after removal of the magnet may induce the partial dispersion of MNPs and thus partially improve hemodynamics. In conclusion, our results revealed the important correlation of local MNP retention and hemodynamic changes in microcirculation, which can be crucial in the application of MNPs for effective targeted therapeutics.
\end{abstract}

Keywords: targeted delivery, magnetic nanoparticles, hemodynamics, microcirculation

\section{Introduction}

Magnetic targeting of therapeutic agents to specific sites in the body is advantageous in that the targeted drugs can be guided to and concentrated at the target area with limited systemic side effects. ${ }^{1,2}$ Magnetic nanoparticles (MNPs) with a magnetite core and organic polymer coating are often used as a drug carrier for this purpose., ${ }^{2,3}$ The MNPs, with characteristics of superparamagnetic magnetization, allow tracking under a magnetic field gradient and maintain stable colloidal suspensions. ${ }^{2}$ These MNPs and the drugs used in magnetic targeting are formulated into a pharmacologically stable composite that is injected into the circulation system and held in position at the target region against hemodynamic force by an external magnetic field. ${ }^{4-13}$
Correspondence: Yunn-Hwa Ma Department of Physiology and Pharmacology, Chang Gung University, 259 Wen-Hwa Ist Road, Kuei-Shan, Tao-Yuan, Taiwan 333, Republic of China $\mathrm{Tel}+\mathrm{I} 886-3-2 \mid \mathrm{I}-8800$ ext 5152

$\mathrm{Fax}+\mid$ 886-3-2II8700

Emailyhma@mail.cgu.edu.tw 
Chemotherapeutic agents are the most studied category of drugs in magnetic targeted delivery to solid tumors in animal models ${ }^{4,7-9,12}$ and in humans, ${ }^{10,11,13}$ primarily due to their highly toxic nature. However, the therapeutic effects vary; this greatly depends on whether the magnetic targeting can be reproducibly achieved. Histological studies have revealed that local retention of MNPs in the tumor vessel or interstitial space of a tumor occur with magnetic targeting, ${ }^{4,9,14-16}$ partly due to magnetic targeting and partly due to the enhanced permeability and retention effect of leaky vessels in tumors. More ${ }^{123}$ I-labeled MNPs have been detected around tumor areas with an external magnet than with no magnet. ${ }^{17}$ Local retention of magnetic liposomes has also been demonstrated in the cranial microvessels of mice using a laser-scanning confocal fluorescence microscope. ${ }^{18}$ In addition, active targeting with magnetic guidance is required for the MNP-drug to exert its anti-tumor effect, ${ }^{4,19}$ implicating the effectiveness of magnetic targeting and its therapeutic potential. Although the response of MNPs in microvessels to external magnets working against hemodynamics has been simulated, ${ }^{20-22}$ their behavior in microvessels in vivo has not been well studied.

MNP administration may cause mechanical occlusion, or embolization, of capillaries under the influence of a magnetic field, as demonstrated in simulated vessels in vitro. ${ }^{20,21}$ Although embolization therapy with MNPs has been tested in the treatment of solid tumors in animal models, presumably by reducing blood supply to the tumors, ${ }^{10,15,23}$ an effective reduction of blood flow in response to MNP administration has not been demonstrated in vivo. In contrast, vascular occlusion may hinder the targeted delivery of drugs using MNPs as carriers; therefore, conditions causing vascular occlusion should be avoided in such an application. Thus, the dose of MNPs and the rate of administration may exert a profound effect on hemodynamics and consequently on therapeutic effects.

MNPs are generally well tolerated with the dose studied

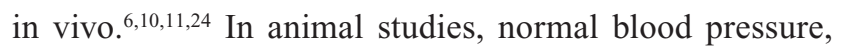
heart rate, and respiratory rate in response to intravenous injection of MNPs was observed; ${ }^{15}$ after administration of MNPs, hemodynamic responses to vasoconstrictors or dilators appeared to be unaltered. ${ }^{15,25}$ However, irreversible magnetic clustering may occur in response to a long enough exposure to a magnetic field, leading to vascular diameter/ hemodynamic changes. ${ }^{15}$ Although MNPs are generally considered superparamagnetic in water, blood components such as plasma proteins may form corona that greatly affect the surface characteristics of the particles in circulation, ${ }^{26}$ allowing the formation of agglomerates in the magnetic field.
To our knowledge, the retention and dispersion of MNPs in microvessels in response to magnetic guidance has not been investigated in vivo.

In this study, we determined the MNP retention profile around a permanent magnet we applied and asked whether MNP retention may affect hemodynamics in the microcirculation of the cremaster muscle. Unlike leaky vessels in tumors, normal, intact microvessels in this preparation allow the direct effect of magnet application on MNP distribution in microcirculation to be determined. Although the cremaster muscle preparation has been suggested as an ideal model to visualize the effects of a magnetic field on the distribution kinetics of MNPs,${ }^{15}$ the traditional setup requires light to pass through the muscle layer for visualization, which hinders the application of a constant magnetic field. The laser speckle perfusion imaging technique used in this study allows the demonstration of two-dimensional real-time changes of tissue perfusion with a magnet placed underneath the muscle. Such techniques have been used to study tissue blood flow since $1982,{ }^{6,27,28}$ but to our knowledge, have not been used to determine the effects of MNP retention in microcirculation. Our results demonstrated a close correlation of the magnetic field, MNP deposits, and hemodynamics in cremaster microcirculation.

\section{Materials and methods Materials}

An MNP with magnetite embedded in a dextran matrix $\left(\mathrm{Nanomag}^{\circledR} \mathrm{D}-\mathrm{COOH}, 250 \mathrm{~nm} ; 10 \mathrm{mg} / \mathrm{mL}\right.$ ), provided as a stable aqueous colloid, was purchased from Micromod Partikeltechnologie GmbH (Rostock, Germany). Neodymium (NdFeB) magnets were purchased from New Favor Industry Co, Ltd (Taiwan). Inactin ${ }^{\circledR}$ (sodium salt of ethyl-[1-methyl-propyl]-malonyl-thio-urea) and heparin (50,000 unit), were purchased from Sigma (St Louis, MO, USA). Hydroxyethyl starch solution $\left(6 \%\right.$, HAES-steril $\left.{ }^{\circledR}\right)$ was purchased from Fresenius Kabi (Germany).

\section{Characterization of MNP}

The particle size was determined by Backman Coulter (N4 plus, Fullerton, CA, USA). The zeta potential of the MNP (Nanomag ${ }^{\circledR} \mathrm{D}-\mathrm{COOH} ; 1 \mathrm{mg} / \mathrm{mL}$, diluted with distilled water) was measured by a Zetasizer (Malvern ZA90, Worcestershire, UK) at $25^{\circ} \mathrm{C}$.

\section{Cremaster muscle preparation}

A rat cremaster microcirculatory preparation was modified from a previously described method. ${ }^{29}$ The protocol was approved by the Institutional Animal Care and Use Committee. Briefly, 
Sprague Dawley rats ( $346 \pm 7 \mathrm{~g}, \mathrm{n}=27$ ) were anesthetized using an intraperitoneal injection of Inactin $(100 \mathrm{mg} / \mathrm{kg})$, followed by a tracheostomy and cannulation of the carotid artery to measure blood pressure. The hair on the lower abdomen and around the scrotum was removed. A rectal temperature probe was inserted to monitor core body temperature, which was maintained at $37^{\circ} \mathrm{C} \pm 1^{\circ} \mathrm{C}$. The urinary bladder was cannulated to ensure a patent urine flow. The cremaster muscle layers on both sides were separated with a bipolar cautery (Union Medical, UM-D30, FL) and the testis was removed. The left iliac artery was cannulated from the left femoral artery in a retrograde manner with PE50 tubing, without interrupting the blood flow to the pudic epigastric artery, a branch of the iliac artery that supplies blood to the cremaster bed (Figure 1A). The right iliac artery was left untouched, allowing the right cremaster muscle to serve as a control. Two small branches of the left iliac artery close to the origin of the pudic epigastric artery were ligated to ensure that most of the injected MNPs reached the cremaster vessels. A mixture of saline and hydroxyethyl starch (1:2) at a rate of $30 \mu \mathrm{L} /$ minute was continuously infused via a catheter in order to maintain a patent catheter for the injection of MNPs (Nanomag ${ }^{\circledR}$ D-COOH; 250 nm, Micromod, Germany). Three to five traction stitches were placed through the distal tip and lateral sides of the cremaster to expose the terminal branches of the microcirculation (Figure 1B). The cremaster muscle was then spread on a homemade stage with a cylindrical $\mathrm{NdFeB}$ magnet $(2.9 \mathrm{kGauss}$, with a radius of $9 \mathrm{~mm}$ and a height of $5 \mathrm{~mm}$ ) placed underneath the left cremaster muscle (Figure 1A), allowing removal of the magnet from below the stage without touching the muscle layer. The maximal magnetic field measured approximately $2.9 \mathrm{kGauss}$ at $5 \mathrm{~mm}$ from the center of the magnet (Figure 1C). The muscle layer was continuously superfused with saline during the experiment. After equilibration, accumulative doses (up to $\sim 80 \mu \mathrm{L}$ total) of MNPs were injected via an injection port of the arterial cannula.

\section{Tissue flow measurement}

The red blood cell velocity/tissue flow of the cremaster muscle was measured with a laser perfusion imager (MoorFLPI, Moor Instruments, England) in a noncontact manner with high spatial and temporal resolution. The setup using a laser diode for illumination provides full field perfusion maps of the cremaster muscle and allows quantification of the arbitrary regions of interest in the left (+MNP) vs right (control) muscle pieces. In addition, simultaneous photos of the preparation using a conventional charge-coupled device camera allow recording of the shape of the muscle
A

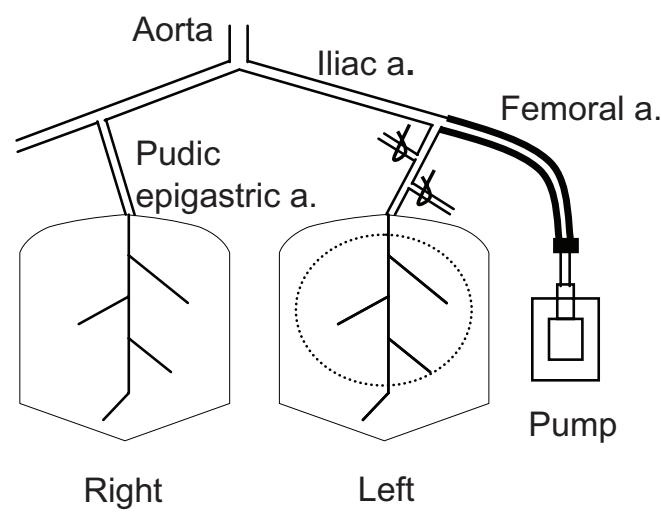

B

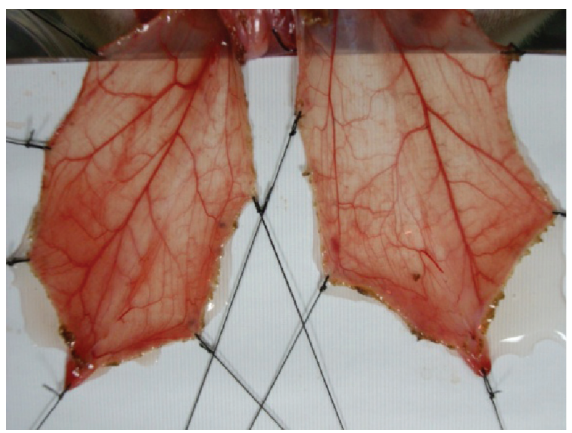

C

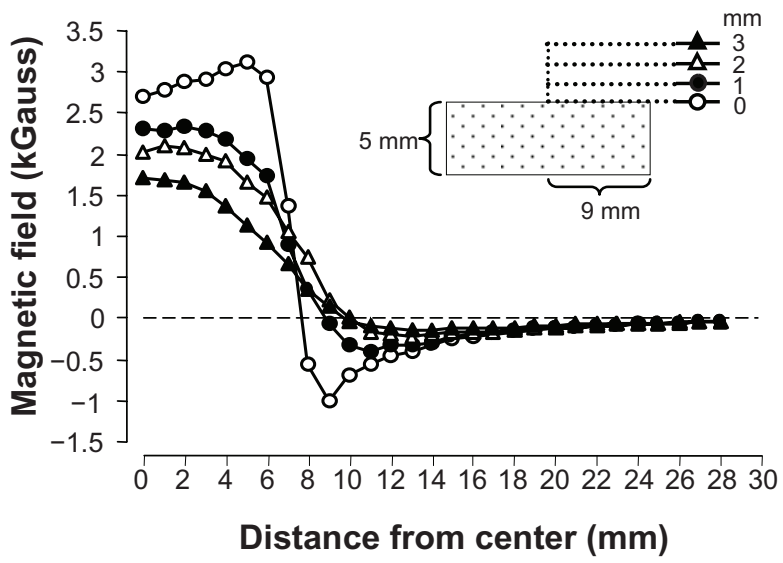

Figure I Rat cremaster muscle preparation for magnetic nanoparticle retention and hemodynamic assessment. Magnetic nanoparticles were injected into the perfusion line that was cannulated into the left femoral artery in a retrograde manner, reaching the pudic epigastric artery, the branch supplying blood to the left cremaster muscle. A neodymium magnet with a radius of $9 \mathrm{~mm}$ was placed underneath the dissected muscle layer on the left, as depicted with a dotted circle, for magnetic nanoparticle capture (A). The image of the muscle pieces of a representative rat was taken using a digital camera (B). The magnetic field of the magnet was measured with a Gauss meter and plotted as a function of distance from the center of the magnet (C)

Note: The tip of the probe of the Gauss meter was placed 0, I, 2, and $3 \mathrm{~mm}$ from the surface along the axial direction of the cylindrical magnet, as indicated.

piece with anatomical and morphological details, as well as major sites of MNP deposits in the vessels of the muscle pieces. Tissue perfusion was measured in a real-time manner and analyzed using MoorFLPI software (version 3.0) and expressed as two-dimensional distributions of hemodynam- 
ics with pseudo-color and percentages of baseline values of entire or partial muscle pieces. In some experiments, $\%$ bin distribution analysis was performed to illustrate quantitative minor changes of the representative muscle piece. At the end of the experiment, the cremaster muscle was harvested after ligation at the base to stop the flow in the artery and vein simultaneously; it was then fixed with paraformaldehyde for further analysis. Histological analysis was conducted with the iron of the magnetite stained with Prussian blue, as previously described. ${ }^{25}$ In some experiments, the muscle layer was weighed, frozen, and stored under $-20^{\circ} \mathrm{C}$ for quantitative analysis of iron in the tissue.

\section{X-ray tomography}

A commercial microcomputed tomography (CT) scanner (SkyScan 1076, Kontich, Belgium) was used in our imaging analysis. SkyScan 1076 is a high-performance micro CT imaging system dedicated to small animal research. An optical charge-coupled device is connected to the system as the photon detector. The sealed x-ray tube generates polychromatic x-ray beams with energies from 20-100 kVp. Various options of spatial resolution in reconstruction images can be selected. The whole imager is housed in a radiation-shielded desktop unit. High-quality images are reconstructed with a high-speed computing system. Cremaster muscle tissue was fixed and stored in paraformaldehyde following the animal experiments. All images were acquired using the settings of $50 \mathrm{kVp}$ and $35 \mu \mathrm{m}$ in spatial resolution. To better display the nanoparticle localization and circulatory distribution within the cremaster muscle, a maximum intensity projection technique was used to create the volumetric images for three-dimensional visualization.

\section{Quantitative analysis of MNP retention in cremaster muscle}

The cremaster muscle was dried at $80^{\circ} \mathrm{C}$ with a vacuum oven for 48 hours, followed by acid digestion using a Multiwave 3000 microwave digestion system (Anton Paar; Austria). The iron content of the samples was measured using an Optima 2100 DV inductively coupled plasma optical emission spectroscopy system (PerkinElmer, USA).

\section{MNP dispersion with ultrasound}

Using a commercially available machine (Sonitron 2000; Richmar, Inola, OK, USA; center frequency $=1 \mathrm{MHz}$ ) with a $10 \mathrm{~mm}$ unfocused probe and coupling gel, ultrasound was irradiated directly to the cremaster bed. Ultrasound exposure was set to a frequency of $1.0 \mathrm{MHz}$, a duty cycle of $20 \%$, a pulse repetition frequency of $20 \mathrm{~Hz}$, and an exposure time of 5 minutes. The exposures began 20 minutes after the removal of the magnet to enhance the dispersion of the MNP pellets in the microvessels of the left cremaster muscle. The pressure at the cremaster bed was measured to be $0.59 \mathrm{MPa}$ using a calibrated polyvinylidene difluoride needle-type hydrophone (Onda, Sunnyvale, CA; calibration range: $50 \mathrm{k}$ to $20 \mathrm{MHz}$ ), which was equivalent to the spatial-peak temporal-average intensity $\left(\mathrm{I}_{\mathrm{SPTA}}\right)$ of $1.05 \mathrm{~W} / \mathrm{cm}^{2}$.

\section{Statistical analysis}

Data are presented as mean $\pm \mathrm{SE}$ and were analyzed using a student's $t$-test or ANOVA with repeated time design followed by a Duncan's post hoc test. Statistical significance was determined as $P<0.05$.

\section{Results}

The diameter of dextran-coated MNP was determined to be $264 \pm 6 \mathrm{~nm}(\mathrm{n}=3)$ by dynamic light scattering; electrophoretic mobility measurements of the MNPs gave a highly negative zeta potential of $-40.1 \pm 0.3 \mathrm{mV}$ at a $\mathrm{pH}$ of $7.9(\mathrm{n}=3)$. To determine whether an NdFeB magnet of 2.9 kGauss may cause effective MNP retention and attenuation of blood flow in cremaster skeletal muscle microcirculation (Figure 1), a laser perfusion imager was used to measure the change of tissue blood flow in response to intra-arterial administration of MNPs.

Figure 2A shows images of representative cremaster skeletal muscle preparation in response to accumulative doses of MNPs, with the magnet placed underneath the left cremaster muscle piece. The pattern of MNP retention appears to be closely associated with the margin of the magnet placed underneath and may occur upstream or downstream of the magnet, but not in the middle, as indicated by the arrowheads. The simultaneous recordings of flux and photo images reveal correlated patterns of flow distribution and MNP retention in the left cremaster muscle preparation. MNP administration and retention caused attenuation of blood flow in the left, but not the right, cremaster muscle compared to that prior to MNP administration. No obvious change in flow was observed in the absence of the magnet (not shown). MNPs at 1,5 , and $10 \mathrm{mg} / \mathrm{kg}$ reduced the perfusion flow by $90 \%, 44 \%$, and $30 \%$, respectively. Prolonged MNP retention ( $\mathrm{p}$ in Figure 2A), recorded 17 minutes after administration of MNPs of $10 \mathrm{mg} / \mathrm{kg}$, further reduced the perfusion to $26 \%$ of the basal level. For this rat, continuous recordings of blood pressure and averaged levels of the perfusion flow are shown in Figure 2B. During the experimental period, the 
A

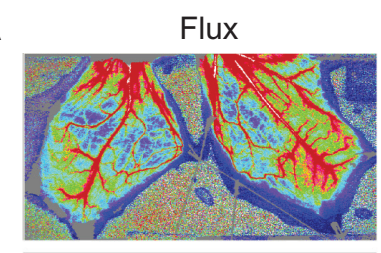

1

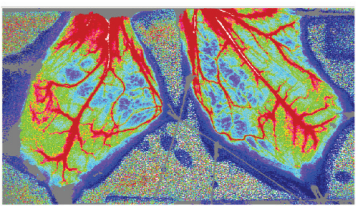

5

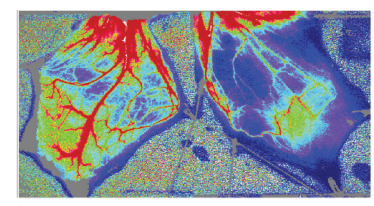

10

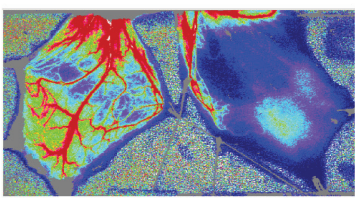

$\mathrm{p}$

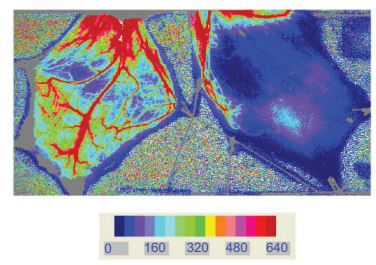

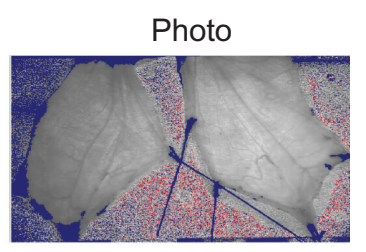
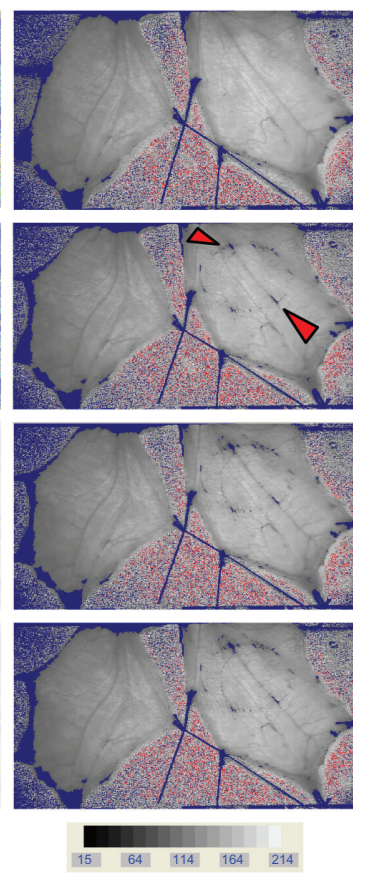

B
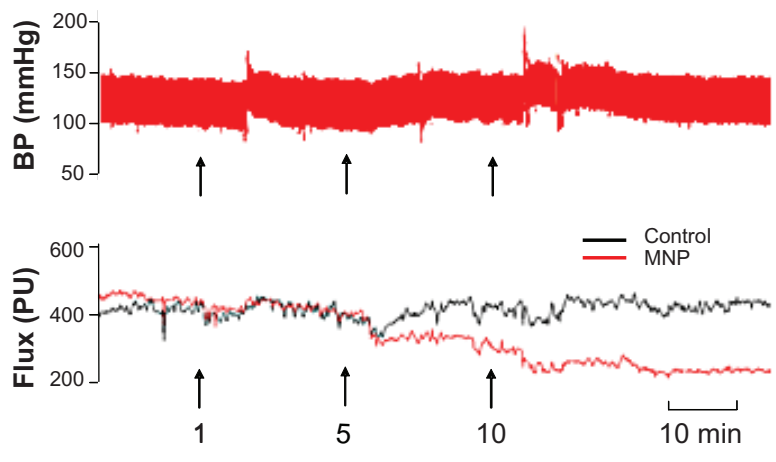

C

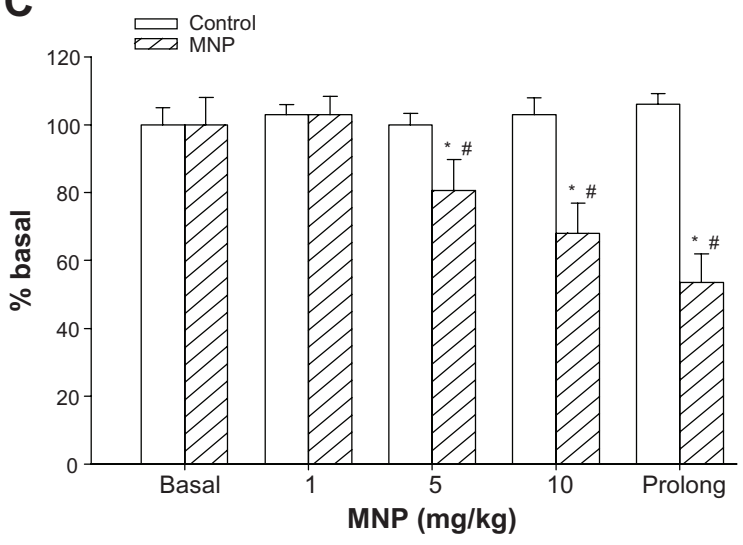

Figure 2 Magnetic nanoparticles (MNPs) attenuated cremaster blood flow in a dose-dependent manner. Accumulative doses of MNPs (I, 5, and I0 mg/kg) were administered via the pudic epigastric artery feeding the left cremaster muscle with a magnet placed underneath. Laser speckle images (Flux) and photo images after each MNP dose or at the end of the experiment for prolonged (P) exposure in a representative rat are illustrated (A); continuous recordings of blood pressure (BP) and the tissue flow (flux; arbitrary unit PU) of the right (control) and the left (MNP) cremaster muscle of this rat are illustrated with time (B).

Notes: The arrowheads in the photo image of $5 \mathrm{mg} / \mathrm{kg}$ MNPs indicate two of the major sites of MNP deposit in vessels. The effect of cremaster flow is summarized as \% basal flow $(C ; n=5)$. $* P<0.05$ compared with the basal value; ${ }^{*} P<0.05$ compared with corresponding control value.

blood pressure and overall blood flow in the right cremaster muscle remained stable with time, whereas blood flow in the left cremaster muscle decreased with accumulative administration of MNPs in the presence of the magnet. The effect of MNP retention on the perfusion flow is summarized in Figure 2C. MNPs at both 5 and $10 \mathrm{mg} / \mathrm{kg}$ significantly reduced basal blood flow by $19 \%$ and $47 \%$, respectively $(\mathrm{n}=5, P<0.05)$. Similar results were obtained when MNPs were administered with $0.5 \%$ bovine serum albumin $(\mathrm{n}=8$, data not shown), suggesting a minor change in the plasma protein composition may not alter the pattern of MNP behavior in response to a magnetic field.

Figure 3 illustrates MNP retention via a variety of methods. Histological analysis with Prussian blue staining demonstrated MNP retention in multiple microvessels of a representative left cremaster muscle preparation subjected to accumulative $10 \mathrm{mg} / \mathrm{kg}$ doses of MNP (Figure 3A). In Figure 3B, MNP distribution patterns in the cremaster muscle preparation of a representative rat are shown with flux, photo, and micro-CT images. In the rat presented in the upper panel of Figure 3B, the magnet underneath the muscle piece caused MNP retention around the edge of the magnet after administration of $10 \mathrm{mg} / \mathrm{kg}$ of MNP. The bright white spot in the micro-CT image on the right side of Figure 3B represents nanoparticle deposit, which is associated with the photo view. As a consequence of particle deposition in the microvessels of the left cremaster muscle, tissue perfusion was attenuated along the edge of the magnet and downstream by $69 \%$; only an $18 \%$ reduction was observed in the right cremaster muscle. It is noted that the blood flow in the artery on the right side of the left cremaster muscle was preserved after MNP administration, suggesting that the flow in the artery on the right margin was not affected by the magnet. Quantitative analysis using inductively coupled plasma optical emission spectroscopy indicated that MNP administration with the magnet almost doubled tissue iron content in the left cremaster muscle piece (Figure 3C). 


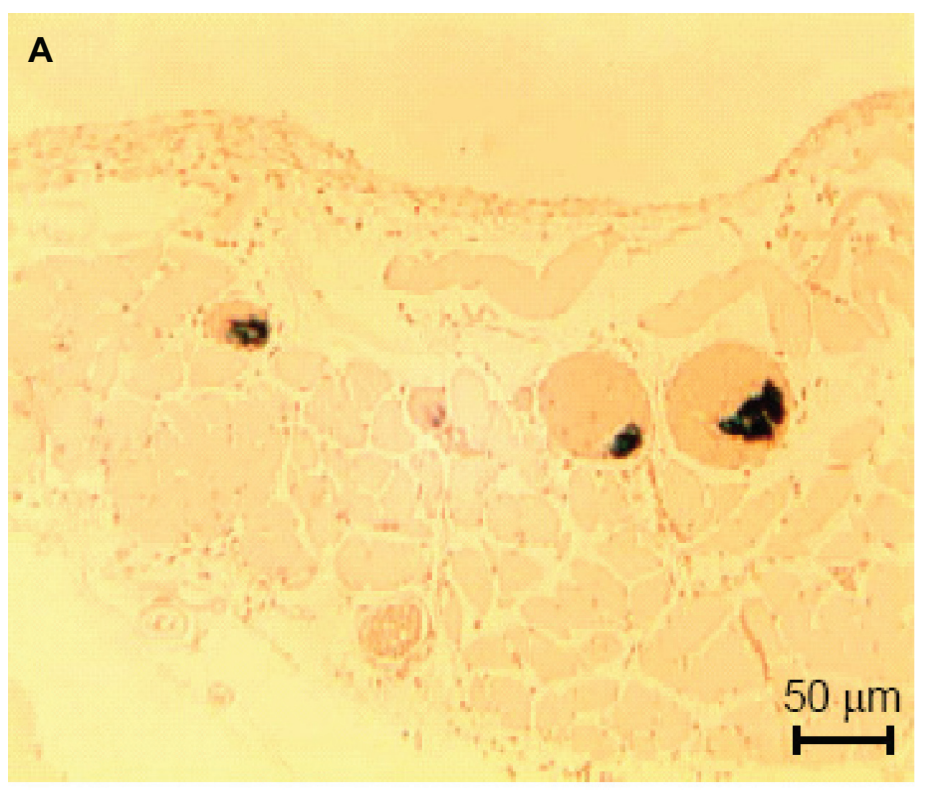

B

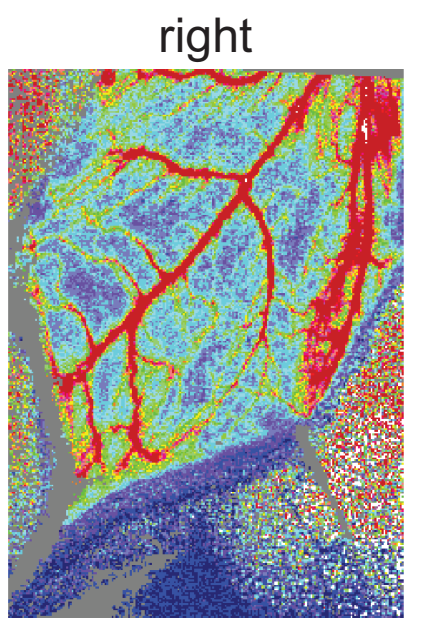

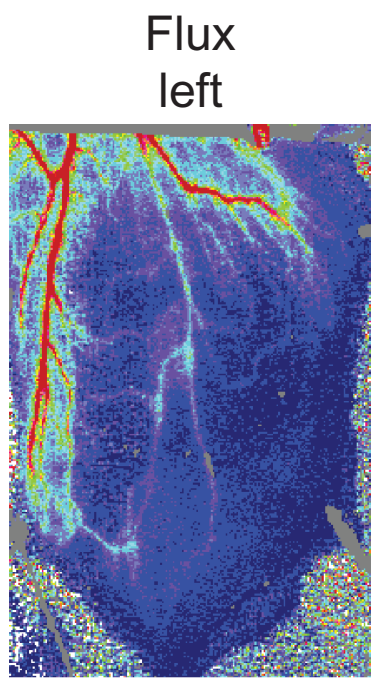

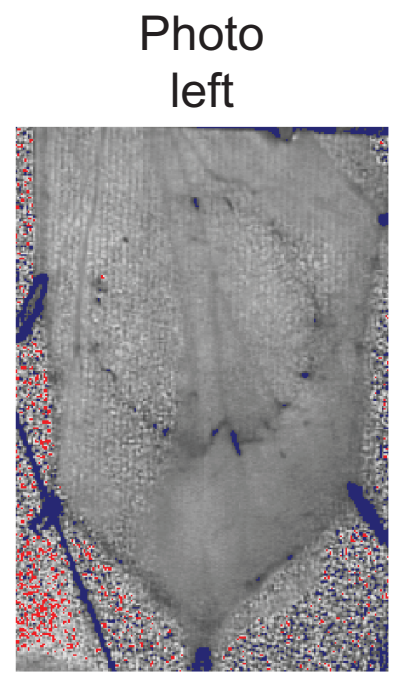

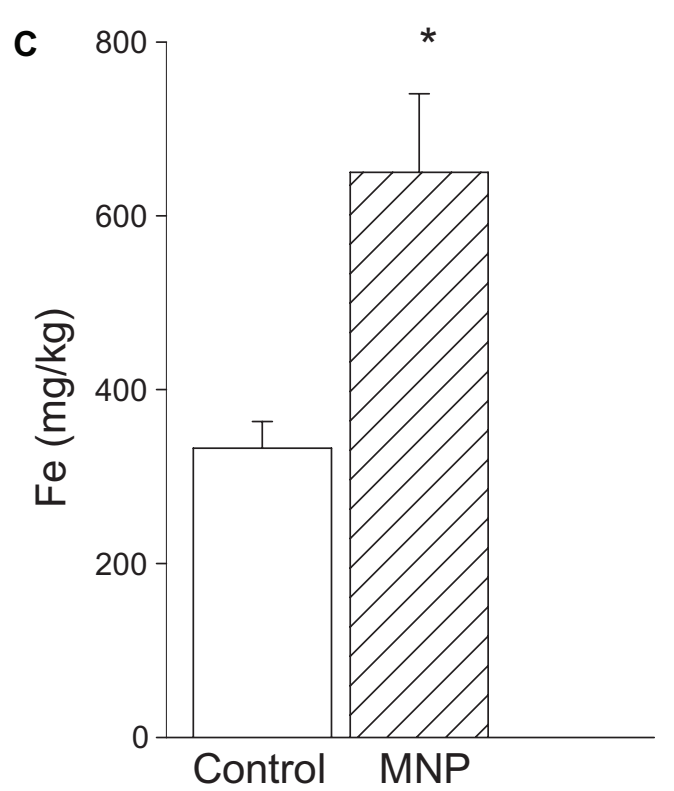

Micro-CT left

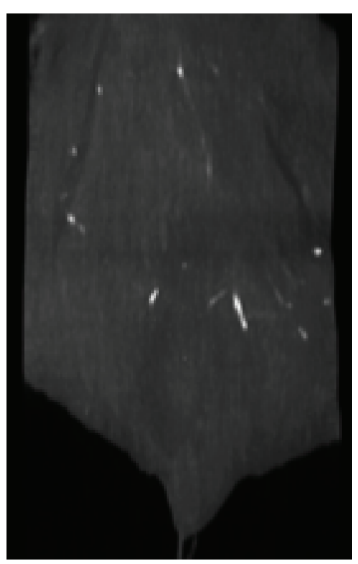

Figure 3 A magnetic field caused magnetic nanoparticle (MNP) retention in cremaster vasculature. In response to accumulative doses of MNPs (I0 mg/kg; i.a.), MNP retention in the cremaster vasculature was demonstrated with Prussian blue staining (A). Both photo images and an image from a micro-CT scanner demonstrate MNP retention in the left cremaster muscle of a representative rat, which is associated with reduced tissue perfusion (B).

Notes: Quantitative analysis of cremaster tissue iron content by inductively coupled plasma optical emission spectroscopy at the end of the experiment is illustrated $(C ; n=5)$. Values are presented as mean $\pm S E$. $* P<0.05$ compared with the control group.

Figure 4 illustrates local retention and dissipation of MNPs in microcirculation observed before and after magnet removal. Administration of MNPs $(5 \mathrm{mg} / \mathrm{kg})$ induced intravascular retention of MNPs in a narrow area along the circumference of the magnet placed underneath the muscle layer, as illustrated in the photo image, which was associated with a $42 \%$ reduction of tissue perfusion. Ten minutes after MNP administration, the magnet was removed. MNP dissipation was observed almost immediately after removal of the magnet, as revealed by the photo image, which is associated with a partial recovery, ie, a 15\% increase in tissue perfusion.
The frequency analysis of the two-dimensional distribution of hemodynamic changes indicated an increase in the lowest perfusion area (the very left two bars in dark blue) of the left, but not right, cremaster muscle piece after MNP administration, presented as \% bin; the lowest perfusion area was reduced more than $50 \%$ after magnet removal, suggesting improved perfusion. The summarized results indicate that total perfusion of the left cremaster muscle attenuated by $32 \%$ vs $37 \%$ after administration of $5 \mathrm{mg} / \mathrm{kg}$ of MNPs vs $10 \mathrm{mg} / \mathrm{kg}$ of MNPs (Figure 5). After magnet removal, left cremaster perfusion increased by $34 \%$ in rats 


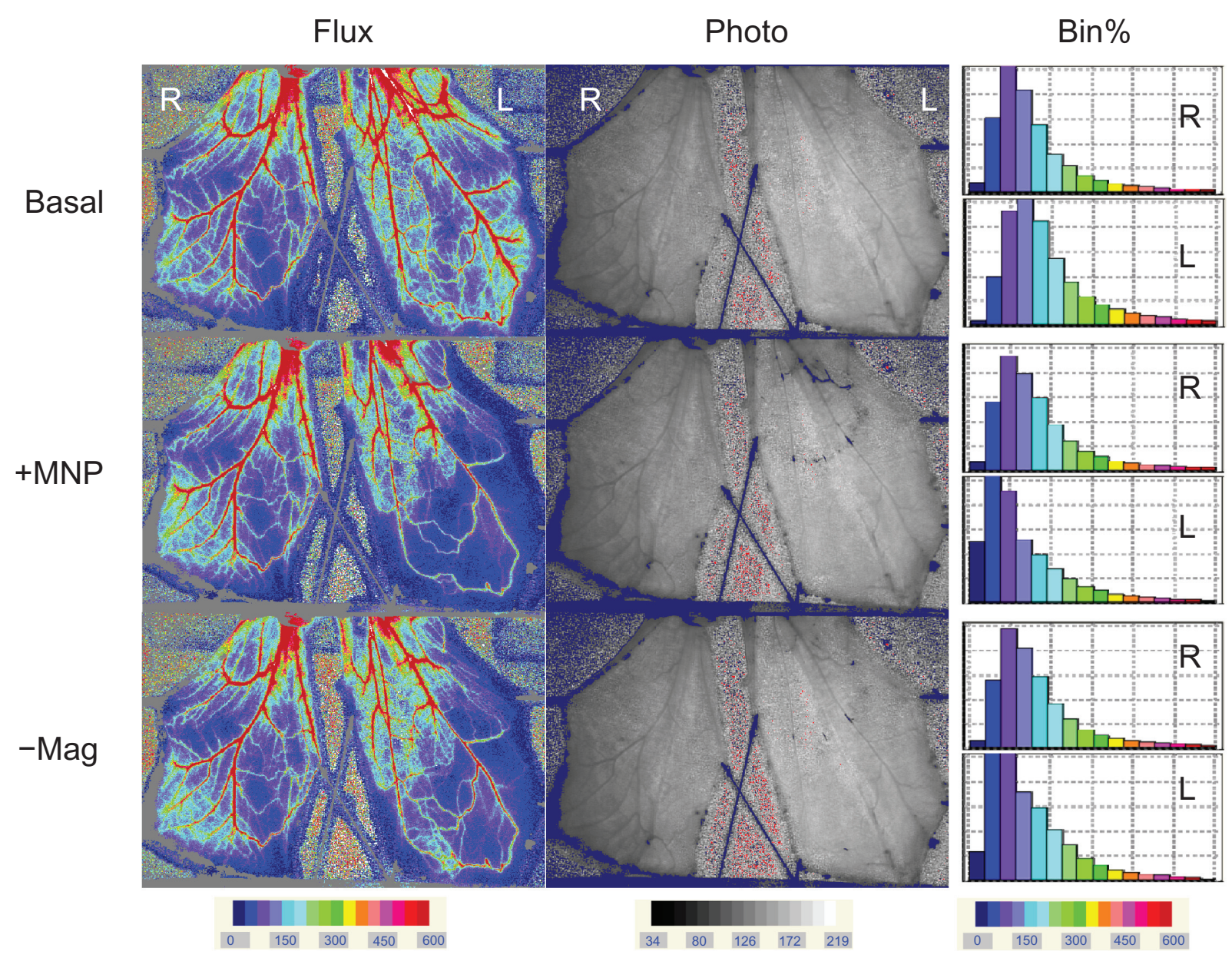

Figure 4 Representative effects of magnet removal-induced MNP dissipation on tissue perfusion in cremaster muscle microcirculation.

Notes: Flux and photo views of both the left $(L)$ vs right $(R)$ cremaster muscle before (Basal) and after MNPs (+MNP; 5 mg/kg) administered to the left cremaster muscle with the magnet underneath the muscle piece are illustrated. Ten minutes after + MNP, the magnet was removed (-Mag); MNP dissipation was recorded approximately 30 minutes after removal of the magnet. Results of the frequency analysis of the perfusion map are illustrated as \% bin in the right panel.

with $5 \mathrm{mg} / \mathrm{kg}$ MNPs $(P<0.05)$. However, removal of the magnet did not cause significant recovery in tissue perfusion in rats with $10 \mathrm{mg} / \mathrm{kg}$ MNPs $(\mathrm{n}=8)$. In experiments with MNPs of $5 \mathrm{mg} / \mathrm{kg}$, tissue perfusion analysis based on areas of upstream, on magnet and downstream suggested that tissue perfusion of only the area upstream of the magnet significantly increased in response to magnet removal (Figure 5B; $P<0.05$ ).

In view of the results from Figures 4 and 5 indicating that removal of the magnet may not restore tissue perfusion, ultrasound was used to determine whether MNPs deposited in the microvessels may be better dispersed. Figure 6 illustrates that intra-arterial administration of MNPs $(10 \mathrm{mg} / \mathrm{kg})$ greatly reduced tissue perfusion by $27 \%$. After 20 minutes, magnet removal did not restore tissue perfusion of the left cremaster muscle. However, ultrasound energy improved tissue perfusion by $13 \%$, which is associated with a minor reduction in the lowest perfusion area.

\section{Discussion}

We demonstrated that magnetic capture of MNPs in the microcirculation may take place at a relatively high local concentration. It is conceivable that MNPs may be captured in microvessels when the magnetic forces dominate the hemodynamic force, as predicted with a simulated capillary. ${ }^{21,30} \mathrm{To}$ our knowledge, the results are the first demonstration of the effect on blood flow of the in vivo administration of MNPs, which can be crucial in future applications. In addition, the pattern of MNP distribution may influence not only downstream hemodynamics, but also the efficacy of the pharmacological agent carried by MNPs in targeted therapy.

In this study, MNP retention associated with magnet placement has been demonstrated using different methods. The photo view of the laser speckle imager allows the observation and recording of MNP distribution patterns simultaneously with the flux view, which reveals hemodynamic information based on red blood cell velocity in 

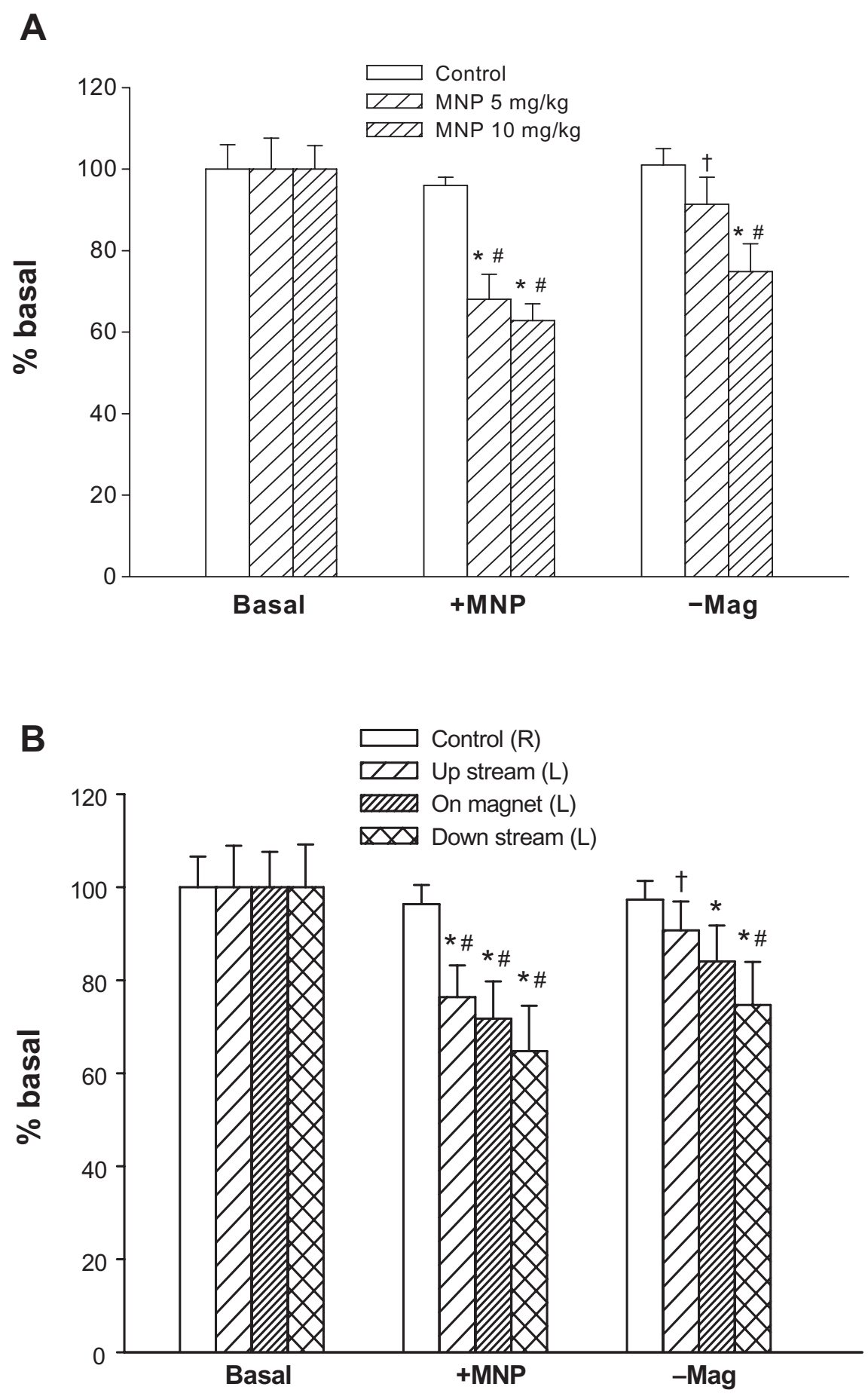

Figure 5 Analysis of hemodynamic effects induced by magnet removal. MNPs at doses of $5(n=1 \mathrm{I})$ or $10(n=8)$ mg/kg were administered to the left cremaster muscle with magnet placement; the right cremaster muscle served as the control. Ten minutes after MNP administration, the magnet was removed. The perfusion of the left cremaster muscle was analyzed as a whole (A) or in parts (B; $5 \mathrm{mg} / \mathrm{kg}$ MNP only) of muscle pieces.

Notes: Relative tissue perfusion on the magnet and upstream/downstream of the magnet were analyzed. $* P<0.05$ compared with basal values of the corresponding group; ${ }^{\#} P<0.05$ compared with control values of the corresponding group; ${ }^{\dagger} P<0.05$ compared with corresponding levels before magnet removal ( $\mathrm{MNP}$ ).

a two-dimensional manner. The distinct patterns of MNP retention reveal strong correlation of the magnet placement and the structure of the vasculature. Since MNP retention occurred along the edge of the magnet, as in the photo view of the laser speckle imager (Figures 2A, 3B, and 4A) and
micro-CT (Figure 3B), and even along the downstream edge of the magnet, as indicated by the lower arrowhead in Figure $2 \mathrm{~A}$, it is anticipated that retention may occur in both arteries and veins. MNPs in the venous deposit might come from a branch upstream of the magnet, which runs through the 


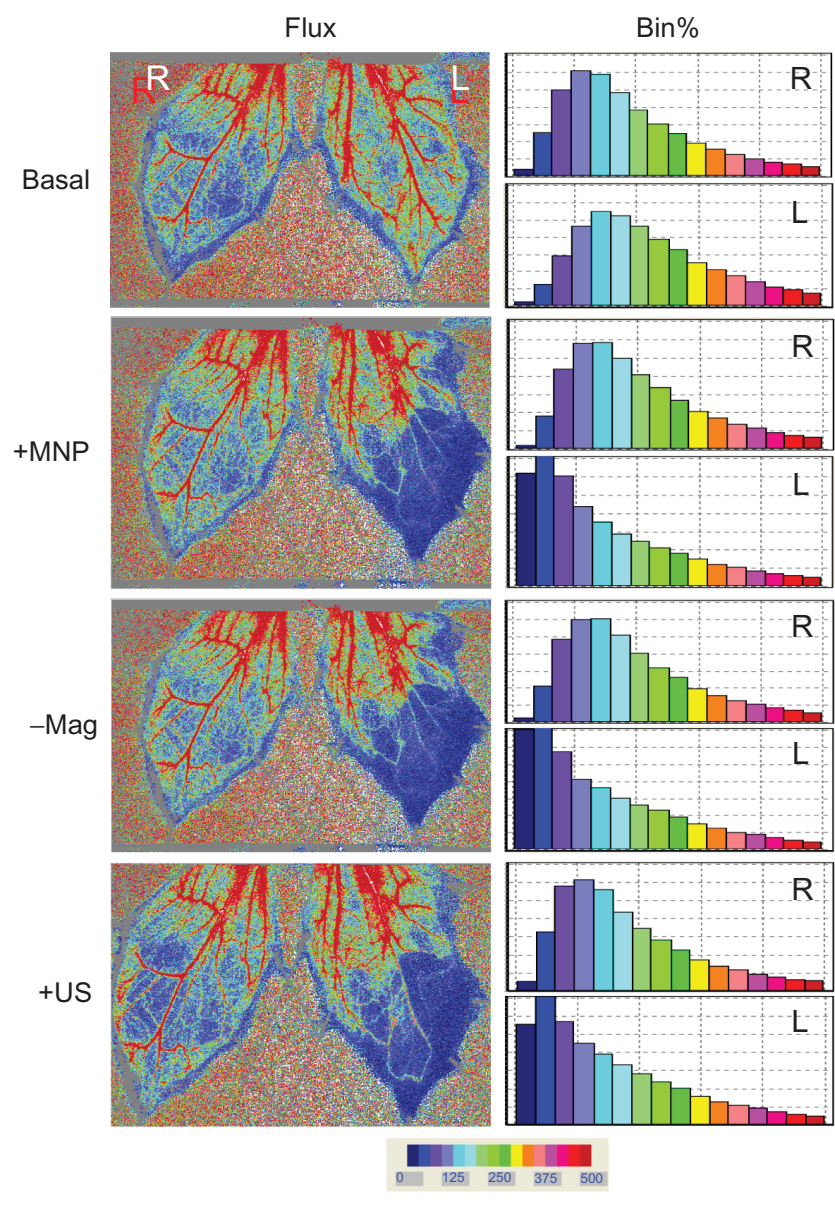

Figure 6 Ultrasound partially restored tissue perfusion of the left cremaster muscle after magnet removal.

Notes: Administration of MNPs (10 mg/kg; +MNP) was followed by magnet removal (-Mag) and application of ultrasound (+US) on the left (L) cremaster muscle piece, with the right $(\mathrm{R})$ cremaster muscle as the control. Results of the frequency analysis of the perfusion map are illustrated as \% bin in the right panel. These results are representative of four experiments.

area outside of that covered by the magnet, and might be captured in the venous system on the way back to the major vein (Figure 7). In this preparation, the veins often run in parallel with the arteries; therefore, MNP capture may occur in adjacent vessels (Figure 3A). We thus propose that MNP retention may occur in the arterial, capillary, and venous systems (Figure 7). Although the magnetic field decreased abruptly on the edge of the magnet, there is still 1 kGauss of magnetic energy at the edge (Figure 1C), which appears to be high enough to overcome flow-induced drag force to cause MNP deposit in these small vessels. However, magnetic field did exert limit, since the arteries close to but not influenced by the magnet preserved its perfusion (Figure 3B). Nevertheless, due to the direction of blood flow in this preparation, tissue perfusion downstream of the area influenced by the magnetic field (even away from the magnetic influence) was prone to decrease in response to MNP retention.

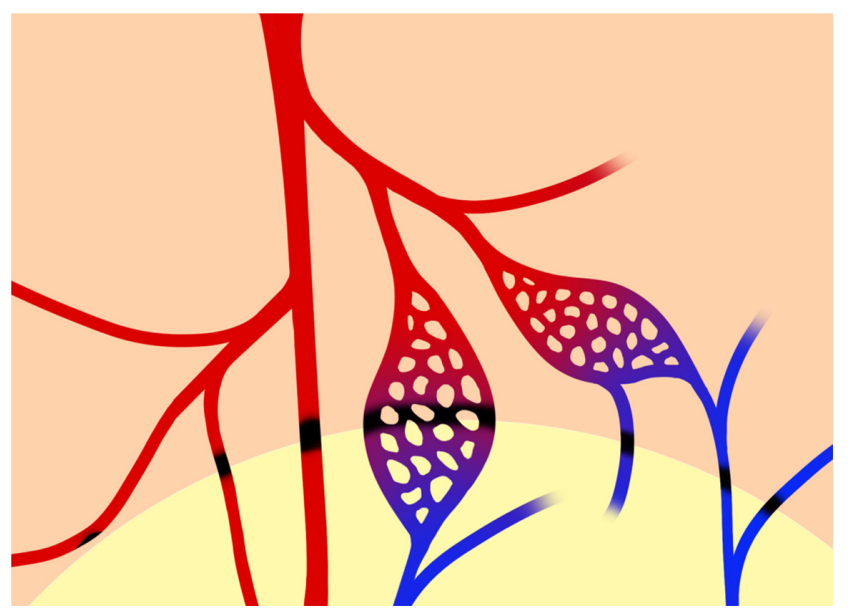

Figure 7 Schematic diagram of MNP retention along the edge of the magnet. Notes: From left to right, MNP retention may occur in the lumen of the 2 nd arteries, the major artery (in red), the capillaries, and the venules (in blue) in the cremaster muscle preparation. MNP deposit (in black) may occur at the margin of the magnet, which may completely or partially block flow in the microvessels. The lower part of the figure with the light background indicates the location of a neodymium magnet underneath the muscle layer.

In contrast, MNP retention in the microvessels of cerebral circulation has been observed without any effect on hemodynamics. ${ }^{18}$ In treating tumors with magnetic targeting, intratumoral embolization by MNPs may ${ }^{10,15}$ or may not ${ }^{4}$ occur. The discrepancy may be due to the dose administered. As higher doses of MNPs were administered in our study, blood flow in the cremaster muscle decreased sequentially (Figure 2), which supports the idea that mechanical occlusion of tumor-feeding vessels may occur after administration of MNPs with high concentrations, causing necrosis and tumor remission. ${ }^{10}$ Although a static magnetic field per se may reduce red blood cell flow in capillaries, ${ }^{31}$ the magnetic field employed in the current study is much lower than the threshold in order for such an effect to occur in mammalian cells. Therefore, the flow reduction response to MNP administration is likely due to mechanical occlusion. This approach may serve as a strategy in the treatment of solid tumors on the body surface. However, in drug targeting, mechanical occlusion may hinder drug distribution in the target site downstream of the occlusion site. Therefore, appropriate doses of MNPs may be essential in targeted drug delivery. ${ }^{10}$

Although the superparamagnetic nature of the magnetite core and the highly negative zeta potential of particles usually allow dissipation and resuspension of MNPs after magnet removal in vitro, ${ }^{6}$ magnet removal did not ensure a quick dispersion of the MNP pellets in vivo, suggesting other factors in circulation, including salt, proteins, and blood cells, may alter the behavior of MNPs in blood vessels. This has 
not really been addressed previously. In addition, a longer application of a magnetic field may facilitate the interaction between MNPs and thus make it more difficult for MNP dispersion to occur after magnet removal. Nevertheless, the application of ultrasound may be a safe way of MNP dispersion in vessels. To our knowledge, the results may be the first demonstration using ultrasound to induce MNP dispersion in vivo, even though the effect of the ultrasound was very limited. The coagulation cascade and/or an inflammatory response may be triggered by reduced flow after MNP retention, resulting in thrombosis and thus irreversible reduction of the flow that hinders recovery from magnet removal or ultrasound application.

Advanced micro-CT systems with high spatial resolution have been widely used in small animal experiments for quantitative imaging in recent years. In the current study, three-dimensional images of MNP distribution were acquired and reconstructed using a commercial microCT scanner. Iron atoms in nanoparticles attenuate larger incident $x$-ray photons than soft tissue in the cremaster muscle. Consequently, a sharp contrast between MNPs and muscle tissue can be seen in micro-CT images where gray levels of nanoparticles are higher than the surrounding muscle tissues. Circulatory distribution patterns of MNPs shown in micro-CT images correspond to external magnet placement, as revealed by the photo images of the laser speckle imager. Therefore, micro-CT imaging may serve as an alternative way to demonstrate the local distribution of MNPs in vivo.

In the targeted delivery of MNP-drug, "passive targeting" by the enhanced permeability and retention effect may occur ${ }^{2}$ as a consequence of the increased vascular permeability and defective lymphatic drainage that are often observed in tumors. ${ }^{32}$ Our results demonstrate that active targeting with magnetic guidance can be achieved in microcirculation, which may contribute, at least in part, to the local retention of MNPs observed around the target area in previous studies. ${ }^{414-16}$ The cremaster muscle preparation may serve as a microcirculation model to study the potential effects of the size or surface characteristics of MNPs in response to a magnetic field in vivo.

\section{Conclusion}

This study demonstrates that magnetic force may readily overcome hemodynamic force and cause vascular retention of MNPs in microcirculation. Magnet-induced MNP retention in microvessels may depend on the topography of magnetic field distribution, and may exert an effect on hemodynamics in a dose-dependent manner. Hemodynamics may be partially restored after the removal of the magnetic influence plus ultrasound application. It is likely that ischemia associated with the reduced flow may be of greater concern than the potential toxicity of MNPs at local sites.

\section{Acknowledgments}

This work was supported by grants from the National Science Council (NSC 982120-M-182-001-), the National Health Research Institute (NHRI-EX9909937EI), and the Healthy Aging Research Program at Chang Gung University (EMRPD1A0841). We thank Professor Li-Man Hung at Chang Gung University for her technical expertise regarding the cremaster muscle preparation and Sonya Y Hsueh for the art work in the schematic diagram.

\section{Disclosure}

The authors report no conflicts of interest in this work.

\section{References}

1. Hafeli UO. Magnetically modulated therapeutic systems. Int J Pharm. 2004;277:19-24.

2. Neuberger T, Schopf B, Hofmann H, Hofmann M, von Rechenberg B. Superparamagnetic nanoparticles for biomedical applications: Possibilities and limitations of a new drug delivery system. $J$ Magn Magn Mater. 2005;293:483-496.

3. Veiseh O, Gunn JW, Zhang M. Design and fabrication of magnetic nanoparticles for targeted drug delivery and imaging. Adv Drug Deliv Rev. 2010;62:284-304.

4. Alexiou C, Arnold W, Klein RJ, et al. Locoregional cancer treatment with magnetic drug targeting. Cancer Res. 2000;60:6641-6648.

5. Alexiou C, Jurgons R, Schmid RJ, et al. Magnetic drug targeting: biodistribution of the magnetic carrier and the chemotherapeutic agent mitoxanthine after locoregional cancer treatment. J Drug Target. 2003;11:139-149.

6. Ma YH, Wu SY, Wu T, Chang YJ, Hua MY, Chen JP. Magnetically targeted thrombolysis with recombinant tissue plasminogen activator bound to polyacrylic acid-coated nanoparticles. Biomaterials. 2009;30: 3343-3351.

7. Hua MY, Liu HL, Yang HW, et al. The effectiveness of a magnetic nanoparticle-based delivery system for BCNU in the treatment of gliomas. Biomaterials. 2011;32:516-527.

8. Alexiou C, Schmid R, Jurgons R, et al. Targeting cancer cells: magnetic nanoparticles as drug carriers. Eur Biophys J. 2006;35:446-450.

9. Yang HW, Hua MY, Liu HL, et al. Self-protecting core-shell magnetic nanoparticles for targeted, traceable, long half-life delivery of BCNU to gliomas. Biomaterials. 2011;32:6523-6532.

10. Lubbe AS, Bergemann C, Huhnt W, et al. Preclinical experiences with magnetic drug targeting: tolerance and efficacy. Cancer Res. 1996;56: 4694-4701.

11. Lubbe AS, Bergemann C, Riess H, et al. Clinical experiences with magnetic drug targeting: a phase I study with 4'-epidoxorubicin in 14 patients with advanced solid tumors. Cancer Res. 1996;56:4686-4693.

12. Widder KJ, Morris RM, Poore GA, Howard DP, Senyei AE. Selective targeting of magnetic albumin microspheres containing lowdose doxorubicin: total remission in Yoshida sarcoma-bearing rats. Eur J Cancer Clin Oncol. 1983;19:135-139. 
13. Wilson MW, Kerlan RK Jr, Fidelman NA, et al. Hepatocellular carcinoma: regional therapy with a magnetic targeted carrier bound to doxorubicin in a dual MR imaging/conventional angiography suite initial experience with four patients. Radiology. 2004;230:287-293.

14. Widder KJ, Marino PA, Morris RM, Howard DP, Poore GA, Senyei AE. Selective targeting of magnetic albumin microspheres to the Yoshida sarcoma: ultrastructural evaluation of microsphere disposition. Eur J Cancer Clin Oncol. 1983;19:141-147.

15. Lubbe AS, Bergemann C, Brock J, McClure DG. Physiological aspects in magnetic drug-targeting. J Magn Magn Mater. 1999;194: 149-155.

16. Yi Y, Kim JH, Kang HW, Oh HS, Kim SW, Seo MH. A polymeric nanoparticle consisting of mPEG-PLA-Toco and PLMA-COONa as a drug carrier: improvements in cellular uptake and biodistribution. Pharm Res. 2005;22:200-208.

17. Alexiou C, Schmidt A, Klein R, Hulin P, Bergemann C, Arnold W. Magnetic drug targeting: biodistribution and dependency on magnetic field strength. J Magn Magn Mater. 2002;252:363-366.

18. Riviere C, Martina MS, Tomita Y, et al. Magnetic targeting of nanometric magnetic fluid loaded liposomes to specific brain intravascular areas: a dynamic imaging study in mice. Radiology. 2007;244:439-448.

19. Liu HL, Hua MY, Yang HW, et al. Magnetic resonance monitoring of focused ultrasound/magnetic nanoparticle targeting delivery of therapeutic agents to the brain. Proc Natl Acad Sci US A. 2010 107:15205-15210.

20. Flores GA, Liu J. In-vitro blockage of a simulated vascular system using magnetorheological fluids as a cancer therapy. Eur Cell Mater. 2002;3:9-11

21. Hallmark B, Darton NJ, Han X, Palit S, Mackley MR, Slater NKH Observation and modelling of capillary flow occlusion resulting from the capture of superparamagnetic nanoparticles in a magnetic field. Chem Eng Sci. 2008;63:3960-3965.
22. Nacev A, Beni C, Shapiro B. Magnetic nanoparticle transport within flowing blood and into surrounding tissue. Nanomedicine. 2010; 5:1459-1466.

23. Turner RD, Rand RW, Bentson JR, Mosso JA. Ferromagnetic silicone necrosis of hypernephromas by selective vascular occlusion to the tumor: a new technique. J Urol. 1975;113:455-459.

24. Weissleder R, Stark DD, Engelstad BL, et al. Superparamagnetic iron oxide: pharmacokinetics and toxicity. Am J Roentgenol. 1989; 152:167-173.

25. Ma YH, Hsu YW, Chang YJ, Hua MY, Chen JP, Wu T. Intra-arterial application of magnetic nanoparticle for targeted thrombolytic therapy: a rat embolic model. J Magn Magn Mater. 2007;311:342-346.

26. Jansch M, Stumpf P, Graf C, Ruhl E, Muller RH. Adsorption kinetics of plasma proteins on ultrasmall superparamagnetic iron oxide (USPIO) nanoparticles. Int J Pharm. In press.

27. Briers JD, Fercher AF. Retinal blood-flow visualization by means of laser speckle photography. Invest Ophthalmol Vis Sci. 1982;22:255-259.

28. Chen JP, Yang PC, Ma YH, Lu YJ. Characterization of chitosan magnetic nanoparticles for in situ delivery of tissue plasminogen activator. Carbohydr Polym. 2011;84:364-372.

29. Anderson GL, Acland RD, Siemionow M, McCabe SJ. Vascular isolation of the rat cremaster muscle. Microvasc Res. 1988;36:56-63.

30. Darton NJ, Hallmark B, Han X, Palit S, Slater NKH, Mackley MR. The in-flow capture of superparamagnetic nanoparticles for targeting therapeutics. Nanomedicine. 2008;4:19-29.

31. Brix G, Strieth S, Strelczyk D, et al. Static magnetic fields affect capillary flow of red blood cells in striated skin muscle. Microcirculation. 2008; $15: 15-26$.

32. Greish K. Enhanced permeability and retention of macromolecular drugs in solid tumors: A royal gate for targeted anticancer nanomedicines. J Drug Target. 2007; 15:457-464.
International Journal of Nanomedicine

\section{Publish your work in this journal}

The International Journal of Nanomedicine is an international, peerreviewed journal focusing on the application of nanotechnology in diagnostics, therapeutics, and drug delivery systems throughou the biomedical field. This journal is indexed on PubMed Central,

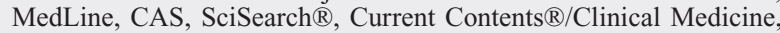

\section{Dovepress}

Journal Citation Reports/Science Edition, EMBase, Scopus and the Elsevier Bibliographic databases. The manuscript management system is completely online and includes a very quick and fair peer-review system, which is all easy to use. Visit http://www.dovepress.com/ testimonials.php to read real quotes from published authors. 